\title{
Experiencias de socialización acerca de amenazas volcánicas en instituciones educativas del municipio de Cumbal (Nariño, Colombia)
}

\author{
Hermes G. Cuaical ${ }^{1 *}$; John J. Sánchez ${ }^{2}$
}

DOI: http://dx.doi.org/10.18273/revbol.v40n3-2018008 (c) (1)

Forma de citar: Cuaical, H.G., y Sánchez, J.J. (2018). Experiencias de socialización acerca de amenazas volcánicas en instituciones educativas del municipio de Cumbal (Nariño, Colombia). Boletín de Geología, 40(3), 181-193. DOI: 10.18273/revbol.v40n3-2018008.

\section{RESUMEN}

Con el objetivo de realizar difusión de información de manera directa sobre volcanes y sus amenazas, se llevaron a cabo diversas actividades de socialización dirigidas a niños y jóvenes en ocho instituciones educativas del municipio de Cumbal (Nariño, Colombia). Las actividades incluyeron conferencias con ejercicios prácticos de apropiación y salidas de campo. Se usaron ayudas audiovisuales con información de varios volcanes del mundo, registros fotográficos de depósitos volcánicos locales y productos científicos como mapas geológicos y columnas estratigráficas. Adicionalmente se realizó un video educativo, orientado por uno de los autores (HGC, quien es originario del municipio de Cumbal y perteneciente a la etnia de los Pastos), en el cual se explican diversos aspectos de vulcanología en un lenguaje accesible a las audiencias. Se observó en los estudiantes y profesores interés en este conocimiento científico, no obstante, el tradicional enfoque etno-pedagógico de estas instituciones en la cuales se enseña que todo lo que rodea es sagrado y místico. Se evidenció facilidad al trabajar con los niños y jóvenes en esta área, pero se reconoce la necesidad de: ampliar la cobertura, mantener la continuidad en actividades y seguir fortaleciendo bases a fin de mejorar la asimilación del conocimiento acerca de volcanes y facilitar la labor de varias instituciones que propenden por la mitigación de los efectos de actividad volcánica.

Palabras clave: Socialización; amenazas volcánicas; riesgos volcánicos; vulcanismo; municipio de Cumbal Colombia.

\section{Awareness experiences on volcanic hazards in educational institutions of Cumbal municipality (Nariño, Colombia)}

\begin{abstract}
With the aim of providing direct information about volcanoes and their hazards, several activities to promote awareness were accomplished targeting children in eight public schools ascribed to the Municipality of Cumbal (State of Nariño in southwestern Colombia). Activities included talks, practical exercises and outings, and used audiovisual aids with information on various volcanoes around the world, such as photographs of volcanic deposits from the region and scientific products like geologic maps and stratigraphic columns. Additionally, an educational video was produced by one of the authors (HGC, who was born in the Municipality of Cumbal and belongs to the Pastos ethnic group) in which a number of aspects of volcano science are explained using simple terms that were accessible to the audiences. The scientific knowledge was of interest to the easy-going students and their instructors despite the strong ethnic-pedagogical tradition in these institutions where instructors teach that everything around is sacred and mystical. The need to widen the coverage is acknowledged as well as the importance of keeping continuity of the activities and strengthening basic concepts to improve both assimilation of knowledge on volcanoes and their hazards and the impact from the activities of various institutions that work toward mitigating the effects of volcanic activity.
\end{abstract}

Keywords: Awareness; volcanic hazards; volcanic risk; volcanism; municipio de Cumbal - Colombia.

${ }^{1}$ Geólogo Independiente. (*) hgcuaicalf@unal.edu.co

${ }^{2}$ Departamento de Geociencias y Medio Ambiente, Facultad de Minas, Universidad Nacional de Colombia, Medellín, Colombia.jjsanchezag@unal.edu.co 


\section{INTRODUCCIÓN}

El municipio de Cumbal, fundado en 1925, se localiza en el Departamento de Nariño y en total la cabecera municipal $\left(0,92^{\circ} \mathrm{N}, 77,8^{\circ} \mathrm{W}, 3050 \mathrm{~m}\right.$. s.n.m. $)$ y áreas rurales superan los 30.000 habitantes. El municipio cuenta con un número limitado de instituciones educativas de niveles primaria y secundaria que atienden las necesidades de aproximadamente 7.000 habitantes en edad escolar (Alcaldía de Cumbal-Nariño, 2012). Parte del municipio de Cumbal se encuentra en el área de influencia del Complejo Volcánico de Cumbal (CVC), ubicado a 12 $\mathrm{km}$ al noroccidente de la cabecera municipal, por lo cual esta experiencia se basa en la socialización de temas relacionados con vulcanismo y amenazas volcánicas en las siguientes ocho instituciones educativas (IE) que fueron visitadas en 2017: IE San Juan de Mayasquer; IE Nuestro Señor del Río; IE Agroecológica Sagrado Corazón de Jesús; IE Técnica Agropecuaria Indígena de Panán; IE Divino Niño Jesús; IE José Antonio Llorente; IE Técnica Agropecuaria Indígena Kumbe; IE Yo Reinaré de Boyera (FIGURA 1).

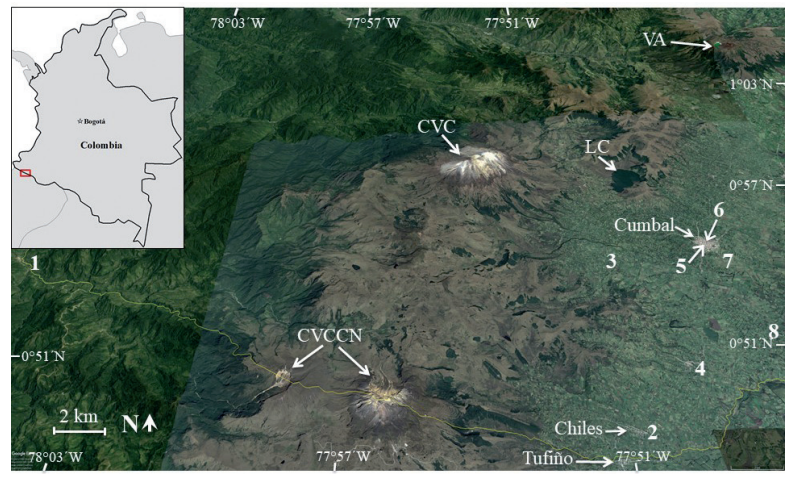

FIGURA 1. Mapa de Colombia con el área de estudio (rectángulo rojo) e imagen del relieve (Google Earth) con la ubicación de sitios mencionados en el texto y las IE visitadas en el municipio de Cumbal. 1=San Juan de Mayasquer; 2=Nuestro Señor del Río; 3=Agroecológica Sagrado Corazón de Jesús; 4= Técnica Agropecuaria Indígena de Panán; 5= Divino Niño Jesús; 6= José Antonio Llorente; 7= Técnica Agropecuaria Indígena Kumbe; $8=$ Yo Reinaré de Boyera. Línea amarilla: frontera colombo-ecuatoriana. VA=Volcán Azufral; $\mathrm{CVC}=$ Complejo Volcánico de Cumbal; LC=Laguna de Cumbal; $\mathrm{CVCCN}=$ Complejo Volcánico Chiles-Cerro Negro.

$\operatorname{Al~CVC~}\left(0,95^{\circ} \mathrm{N}, 77,87^{\circ} \mathrm{W}, 4.764 \mathrm{~m}\right)$ se tiene acceso por una carretera que conduce desde el municipio de Cumbal, pasa por el sector La Ortiga y continúa en un camino de herradura (FIGURA 1). El CVC está conformado por dos volcanes compuestos contiguos y activos de edad PlioPleistoceno-Holoceno: Mundo Nuevo, con un cráter de $200 \mathrm{~m}$ de diámetro mayor, y La Plazuela, un cráter de 600 $\mathrm{m}$ de diámetro (FIGURA 2). El CVC se emplazó sobre basamento Cretácico, conformado por rocas volcánicas con afinidad oceánica y sedimentarias de origen marino. Sus productos más nuevos son flujos de lava escoriáceos, en bloques y masivos superpuestos, flujos piroclásticos de diversos tipos, flujos de escombros y depósitos de caídas piroclásticas que recubren toda el área. Algunas muestras analizadas de estos productos se han clasificado como andesitas de dos piroxenos (INGEOMINAS, 2000).

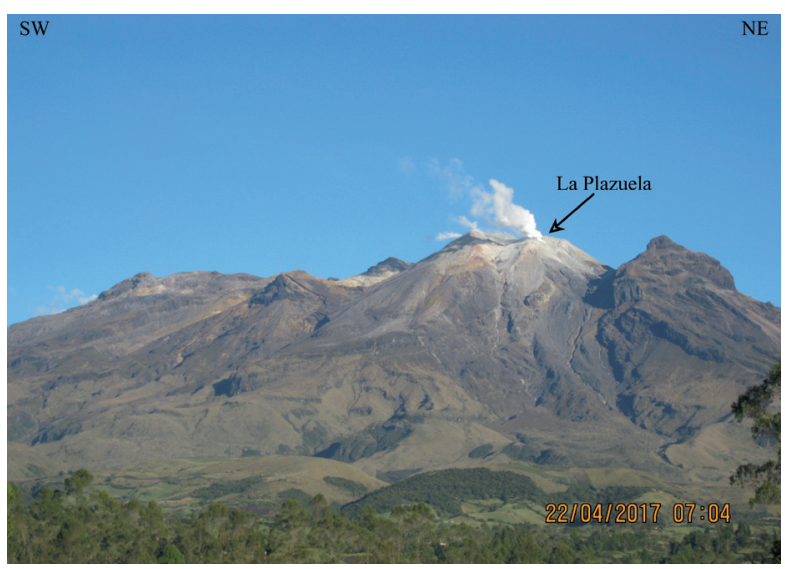

FIGURA 2. Fotografía del Complejo Volcánico de Cumbal. Se observa el cráter La Plazuela.

Otras áreas del municipio se encuentran en la región de influencia del Complejo Volcánico Chiles-Cerro Negro $(\mathrm{CVCCN})\left(0,82^{\circ} \mathrm{N}, 77,94^{\circ} \mathrm{W}\right)$, conformado por los volcanes Chiles (4.698 m.s.n.m.) y Cerro Negro de Mayasquer (4.470 m.s.n.m.) (INGEOMINAS, 2000), que se localizan en la frontera colombo-ecuatoriana con acceso por dos carreteras principales: una que parte desde la población de Chiles hasta Tiuquer (Colombia), y otra desde la población de Tufiño hasta Maldonado (Ecuador). Se trata de dos volcanes compuestos activos contiguos, cuyos cráteres tienen diámetros de 1,0 y 1,8 $\mathrm{km}$, respectivamente. El río Carchi, que en parte es la frontera natural entre los dos países, tiene su cabecera en el volcán Chiles (FIGURAS 3 y 4).

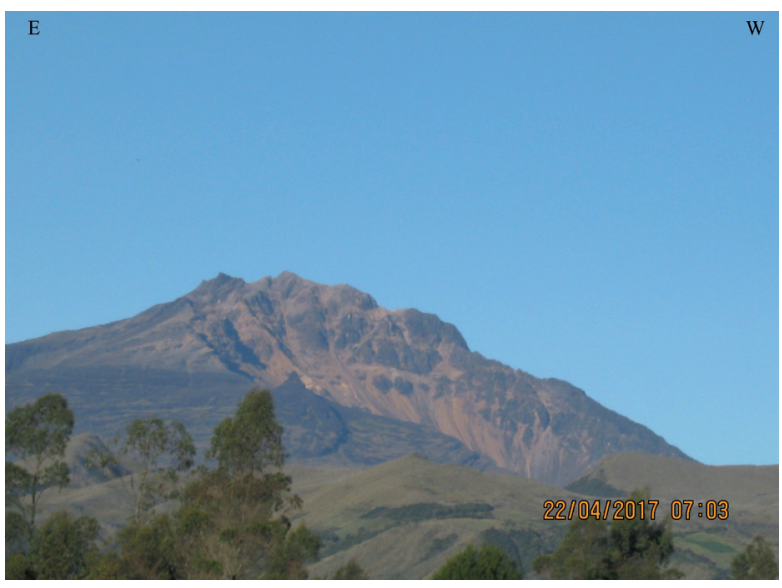

FIGURA 3. Fotografía del volcán Chiles. 


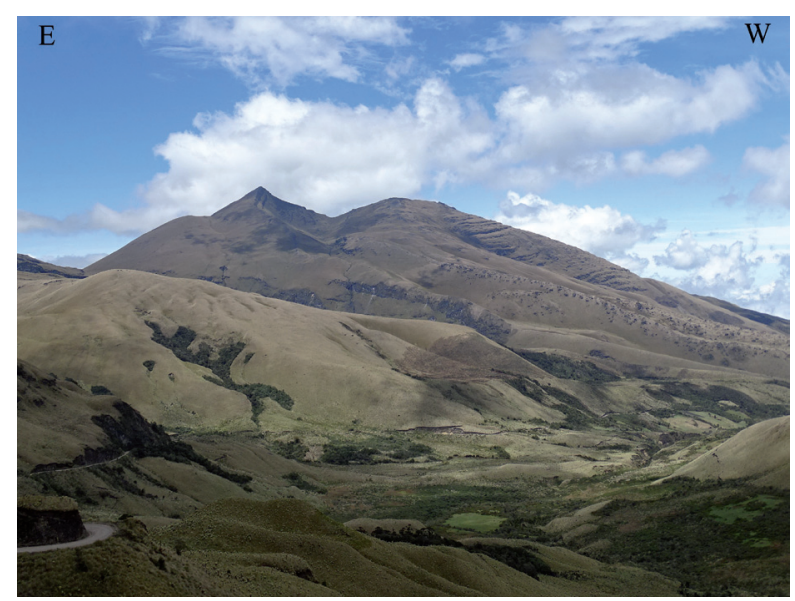

FIGURA 4. Fotografía del volcán Cerro Negro de Mayasquer.
El magmatismo que eventualmente dio origen a estos volcanes quizá fue facilitado por intersecciones de la falla Chiles-Cerro Negro, con las fallas Chiles-Cumbal y Cerro Negro-Nasate (FIGURA 5). Los edificios volcánicos se construyeron sobre un basamento representado por una secuencia potente, que de techo a base incluye lavas pliocénicas, rocas volcanosedimentarias de la Formación Nariño, rocas de los grupos Dagua y Diabásico y rocas metamórficas del Grupo Cajamarca (Velásquez y Parra, 2002). El edifico actual del volcán Chiles está conformado fundamentalmente por flujos de lava y el volcán Cerro Negro por flujos de lava y depósitos de piroclásticos. De acuerdo con análisis químicos y petrográficos, los productos del CVCCN se clasifican como andesitas de dos piroxenos y andesitas olivínicas (INGEOMINAS, 2000) (FIGURA 5).

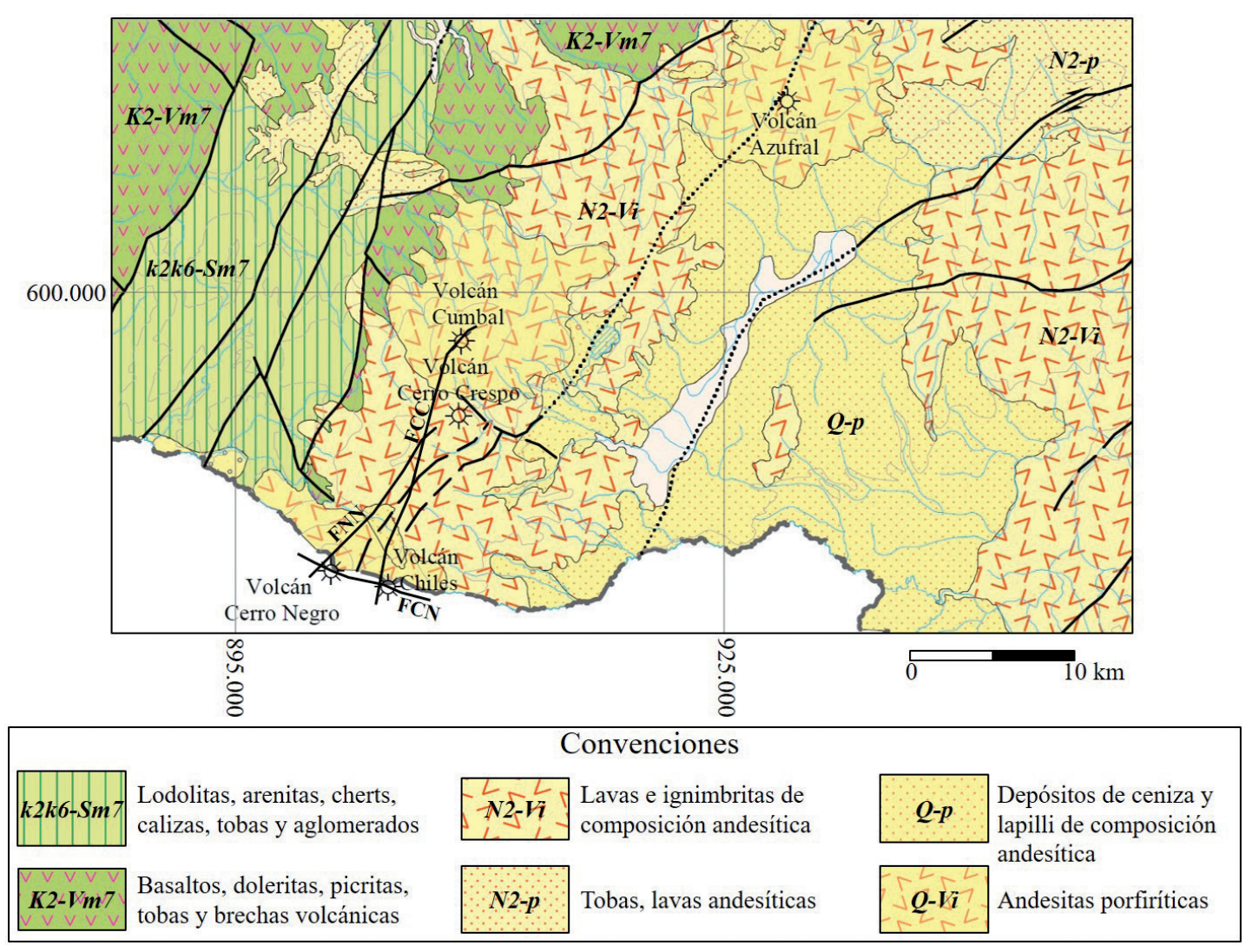

FIGURA 5. Mapa geológico regional en zona de confluencia del Complejo Volcánico de Cumbal (CVC) y Complejo Volcánico Chiles-Cerro, Negro (CVCCN). FCN: Falla Chiles-Cerro Negro, FCC: Falla Chiles-Cumbal, FNN: Falla Cerro Negro-Nasate. Modificado de Gómez et al. (2015).

\section{Algunos antecedentes de interacciones volcanes- humanidad}

A continuación, se presenta una síntesis de experiencias en otros países acerca de interacciones volcaneshumanidad, y la interacción entre comunidades, científicos y tomadores de decisiones, para dar bases sobre las cuales se puedan establecer similitudes o diferencias con situaciones en Colombia.

Uno de los mayores desastres volcánicos sucedió luego de la erupción del Monte Pelée (Martinica) en 1902. Durante las noches previas, los sonidos y la vibración del suelo perturbaron a los habitantes, pero 
el gobernador Louis Mouttet, restó importancia a estos hechos y a la información sobre la creciente actividad volcánica (Scarth, 2002). La sugerencia de evacuación en Martinica planteó tres problemas interrelacionados: volcánicos, políticos y psicológicos (Scarth, 2002; Lécuyer y Cheminée, 2010), de modo similar a lo sucedido en Colombia durante 1984-1985 cuando la actividad del volcán Nevado del Ruiz llamó la atención y generó temor en la región, pero según estudios, el proceso de toma de decisiones fue lento e ineficaz (Hall, 1990; Voight, 1990; El Diario, 2015). En Martinica durante la erupción de Mount Pelée, la cifra de fatalidades se estimó en 29.000 personas, incluyendo los habitantes de Saint Pierre, que era el centro social, comercial e industrial en la isla (Fort-de-France, capital administrativa, era la segunda ciudad más grande). Luego de la erupción, Fortde-France pasó a ser el único centro de importancia en la isla y concentró la mayor parte del poder económico.

La erupción de El Chichón, México en 1982, ocasionó la peor catástrofe volcánica en tiempos históricos mexicanos (Espíndola et al., 2000; Macías et al., 2003). Los depósitos de las erupciones de hace 1250 y 2500 años contienen fragmentos de cerámica, sugiriendo interrelaciones entre asentamientos humanos y actividad volcánica. Los indígenas Zoque, dedicados a la ganadería y la siembra, se negaron a evacuar por temor al vandalismo y también manifestaron el estar acostumbrados a convivir con el volcán. En este caso, los indígenas se guiaban por pensamientos míticos y religiosos, una cosmovisión que no incluía razonamiento de tipo científico. Las caídas de ceniza y flujos piroclásticos de dos erupciones explosivas causaron cerca de 2.000 víctimas fatales, más de 20.000 damnificados, y considerables pérdidas materiales (Macías et al., 2003; Espíndola et al., 2000; Tilling, 2009).

Es importante el caso del volcán Turrialba (Costa Rica), donde los estudios y la zonificación de amenazas se usaron para planificar el uso del suelo. En la zona de amenaza alta, se sugirieron áreas de pastoreo y cultivos y se recomendó, que la única actividad económica allí, fuera el turismo en condiciones adecuadas. Se sugirió uso forestal y vivienda de baja densidad para el área con radio de $5 \mathrm{~km}$ alrededor del cráter y se especificó que los centros poblados cuenten con planes de respuestas rápidas ante alertas (Soto, 2012).

La reactivación del Nevado del Ruiz y la destrucción de Armero, siguen citados como el desastre natural más fatal en Colombia. Una erupción explosiva fundió parte de la cubierta de nieve y hielo en la cima del volcán, donde nacen los ríos Gualí, Azufrado, Lagunillas, Molinos y Nereidas. La ciudad de Armero fue arrasada por lahares que recorrieron los ríos Azufrado y Lagunillas mientras que los ríos Molinos y Nereidas encauzaron lahares al sistema río Claro-río Chinchiná, y alcanzaron la periferia de la ciudad de Chinchiná (Pierson et al., 1990). En Armero las víctimas fatales superarían 23.000 personas y en Chinchiná alrededor de 2.000. Las pérdidas económicas se estimaron en 211,8 millones de dólares, de acuerdo con cifras del Programa de las Naciones Unidas para el Desarrollo (PNUD). También ocurrieron lahares en 1595 y 1845 , este último causando también alrededor de 1.000 fatalidades (Mojica et al., 1985). Los hechos del 13 de noviembre de 1985 sucedieron a pesar de señales de reactivación desde 1984, de recomendaciones de expertos nacionales e internacionales, y a pesar del mapa de amenaza volcánica oficializado el 7 de octubre de 1985, en el cual Armero se reconocía en zona de amenaza alta por lahares (Parra et al., 1986; Latorre, 1987; Hall, 1990; Parra y Cepeda, 1990; INGEOMINAS, 2000).

No obstante, la dura lección generó acciones positivas: antes de 1985, Colombia no tenía estructura institucional para afrontar situaciones desastrosas, y fue solo a partir de la catástrofe, cuando se inició el proceso normativo y de conocimiento del riesgo. Se creó el Sistema Nacional de Prevención y Atención de Desastres (SNPAD) como red institucional para el cumplimiento de esta función y, posteriormente, la Unidad Nacional para la Gestión del Riesgo de Desastres (UNGRD) (decreto número 4147 del 3 de noviembre de 2011), para gestionar el riesgo de desastres atendiendo políticas de desarrollo sostenible, y coordinar el funcionamiento y el desarrollo del SNPAD (UNGRD \& Presidencia de la República, 2015).

\section{Experiencias en socialización de amenazas volcánicas}

En años recientes muchos países han alcanzado, con trabajo coordinado y aproximaciones multidisciplinarias, un progreso importante en lo relacionado con la ciencia de los volcanes, monitoreo de actividad volcánica, monitoreo de actividad sísmica, y prevención de desastres. Parte de las actividades de prevención se han enfocado en la producción de materiales didácticos para diversas audiencias y la educación con participación activa de las comunidades. Por ejemplo, en Perú, donde la actividad en volcanes como Ubinas o El Misti representa amenaza y riesgo para decenas de miles de habitantes, los libros sobre volcanes y sus amenazas, escritos para una amplia audiencia, son considerados una contribución importante para preparar a la población (Macedo, 2012). En Colombia, una aproximación a la producción de cartillas bilingües para audiencias 
infantiles (Gil y Sánchez, 2014), puede ser útil como una componente en campañas educativas acerca de amenazas volcánicas. En actividades con participación activa de los habitantes de áreas volcánicas, el Servicio Geológico Colombiano-Observatorio Vulcanológico y Sismológico de Pasto (SGC-OVSP), ha llevado a cabo acciones concretas de socialización concerniente a varios volcanes de la región de Nariño, entre ellos: Azufral, Cumbal y Chiles-Cerro Negro de Mayasquer. En relación con las experiencias en el municipio de Cumbal, se han adelantado actividades particularmente con la niñez y la juventud (Gómez y Narváez, 2017). A continuación se resumen algunas de estas acciones: 1) noviembre de 2013: se realizó el evento "Segunda bienal nacional de niños, niñas y jóvenes que viven en zonas de riesgo volcánico", en San Juan de Pasto y con la participación de la Institución Educativa Los Andes de Cuaical; 2) agosto de 2014: a raíz de una reunión entre la comunidad y el Ministerio del Interior, surgieron propuestas como la creación de una litoteca y una columna estratigráfica, que actualmente se pueden apreciar en la Institución Los Andes de Cuaical; 3) mayo 25-29 de 2015: el SGC-OVSP realizó la actividad de "Observatorio Itinerante" dirigida hacia la comunidad estudiantil, durante la cual se desarrollaron actividades de estudio como: sismología, deformación, geoquímica, entre otras; 4) agosto de 2015: se designa al municipio de Cumbal como centro para el desarrollo de estrategias de comunicación del riesgo volcánico, mediante un acuerdo firmado entre el SGC, UNGRD y el Observatorio Colombiano de Ciencia y Tecnología (OCyT). Se han realizado siete talleres en total que han tenido sede en instituciones de la cabecera municipal de Cumbal y la Casa de Cabildo de Chiles, liderados por el personal del OCyT y del SGC. Dentro de las actividades que más llaman la atención a los participantes destaca la parte experimental que apoya la asimilación de los conceptos vulcanológicos.

Como contribución y complemento a las acciones mencionadas, este trabajo reporta la experiencia de actividades pedagógicas en ocho instituciones educativas (IE) del municipio de Cumbal entre febrero y abril de 2017, con los objetivos particulares de socializar conocimiento acerca de amenazas por vulcanismo y exponer opiniones contrastantes entre la geología y la cosmovisión de los niños y jóvenes indígenas acerca del territorio.

\section{METODOLOGÍA}

La metodología de este trabajo se enmarca dentro de la sistematización de experiencias, que es la interpretación de una o varias experiencias en términos del proceso vivido y los factores que intervinieron. A partir de la sistematización de experiencias se conoce y aprende significativamente para producir transformaciones a futuro (Jara, 2008; Bermúdez, 2018). La idea de socializar conocimiento acerca de volcanes surgió debido a la afinidad con la comunidad del municipio de Cumbal, ya que uno de los autores (HGC) es miembro de la comunidad indígena y conoce bien muchos aspectos de la vida cotidiana, las tradiciones ancestrales y la cosmovisión de los habitantes de la cabecera municipal y áreas rurales. A continuación, se describen las fases para la realización de las socializaciones en las instituciones educativas.

(a) Fase 1: Ubicación y selección de las IE adscritas al municipio de Cumbal. Durante esta fase se realizaron salidas de campo y visitas para contactar a educadores y directivos. Bajo criterios de interés y máxima cobertura espacial, se seleccionaron ocho instituciones de nivel primaria y secundaria. Seis instituciones prestan sus servicios en áreas rurales (incluyendo los corregimientos/resguardos indígenas de Panán y Chiles) y dos están ubicadas en la cabecera municipal de Cumbal (FIGURA 1).

(b) Fase 2: Planeación de la actividad pedagógica. En esta fase se informó por escrito a los rectores de cada IE acerca de la actividad a desarrollar (título de la conferencia, objetivo general, objetivos específicos, tabla de contenido, una breve presentación personal de HGC y un resumen de las actividades propuestas), de este modo se suscitó retroalimentación a través de recomendaciones, sugerencias, comentarios, y se establecieron fechas para el desarrollo de las visitas. En cada caso se recomendó la selección previa de dos o tres representantes por cada curso y se solicitó destinar algunos recursos logísticos como: proyector, aula, y contar con un tiempo de dos horas y media ante los grupos.

(c) Fase 3: selección y preparación de material e información técnica. En esta fase se realizó la preparación material didáctico para una conferencia y una actividad llamada "taller práctico". Se seleccionaron temas básicos (que no pueden faltar al hablar sobre volcanes, por ejemplo "estructura interna de la Tierra" y “tectónica de placas"); temas específicos que le daban nivel a la conferencia y requerían poner en contexto lo aprendido en la parte básica (por ejemplo, "volcanes activos de Colombia" o "procesos volcánicos"); y temas opcionales para ampliar y profundizar si el tiempo lo permitía (por ejemplo: 
"fuentes del magma" o "casos de estudio de catástrofes por vulcanismo"). También se seleccionó material audiovisual de apoyo: fotografías, mapas y columnas estratigráficas. Se estimaron de manera aproximada las duraciones de cada parte de la conferencia y del taller.

(d) Fase 4: realización de conferencia y taller. Estas actividades, con duración (planeada) de dos horas y media, fueron divididas en cuatro partes. Durante la primera se definieron conceptos como: vulcanología, volcán, amenaza y riesgo volcánico, ceniza, lapilli, lava, tipos de erupciones, pómez, columnas y nubes eruptivas, gases volcánicos, flujos y domos de lava, flujos piroclásticos o flujos calientes de ceniza, deslizamientos o derrumbes volcánicos, lahares. En la segunda parte se describen los volcanes Cumbal, Chiles, Cerro Negro de Mayasquer, Cerro Canguil, Cerro Crespo-Nasate y Cerro Colorado. Aquí se hace referencia a la geología en el territorio del municipio de Cumbal, que comprende unidades con edades entre el Cretácico y el Pleistoceno - Holoceno, como los depósitos de morrenas suprayacidos por capas de ceniza y lapilli con continuidad lateral bastante amplia observable en varios sitios, por ejemplo, en los sectores Guairés Alto, Tolas, y desde el punto donde la quebrada Pistejo cruza el camino hasta Guel. Se preparó un registro fotográfico previo de estos sitios.

En la tercera parte se comentaron experiencias de las comunidades, instituciones y gobiernos de Colombia y otros países frente a la actividad volcánica. Se discutieron, por ejemplo, las experiencias de la comunidad indígena Zoque aledaña al volcán Chichón, de la población cercana al volcán Cotopaxi en Ecuador, y de la comunidad indígena Nasa cercana al volcán Nevado del Huila, entre otras. En cuanto a la institucionalidad se mencionó la UNGRD y demás instancias departamentales y locales que se encargan de atender y mitigar los efectos de procesos geológicos amenazantes. En la cuarta parte se hizo mención de erupciones volcánicas significativas, entre ellas: Nevado del Ruiz, Vesubio, Etna, Tambora, etc. Como apoyos audiovisuales, se usaron algunos mapas en tamaño póster, por ejemplo: la Tierra con las diferentes placas litosféricas, columnas estratigráficas relacionadas con los depósitos volcánicos regionales, estructura de un volcán, tabla del tiempo geológico, mapas de amenaza volcánica del territorio, mapas topográficos y los mapas geológicos 447 y 447 bis (Velásquez y Parra, 2002). Durante todas las fases de la experiencia, se tuvieron en cuenta los aspectos éticos relacionados a la mención de créditos de autores de los diferentes materiales didácticos, consideraciones ambientales, y ante todo la manifestación explícita durante cada socialización del respeto por la cosmovisión y el conocimiento ancestral. La FIGURA 6 resume las fases y acciones adelantadas para la realización de las socializaciones y en la TABLA 1 se sintetizan aspectos relevantes de todas las visitas de socialización realizadas.

\section{RESULTADOS}

Aparte de la satisfacción de contribuir con aspectos educativos de la comunidad escolar del municipio de Cumbal, se obtuvieron los siguientes resultados: aprendizaje por parte de los autores acerca de la importancia del conocimiento ancestral y las ideas sobre cosmovisión en relación con la aceptación de propuestas de investigación en la región; cambio parcial de la visión acerca del fenómeno volcánico, ya que los estudiantes e instructores manifestaron que era importante la exposición a varios temas científicos de los volcanes porque ayuda a complementar y entender mejor este aspecto del ambiente; acercamiento con los miembros de cada IE; formación de red de nuevos contactos, incluso con potencial de vinculación laboral para HGC. Luego de la interacción con los participantes de las socializaciones iniciales, se usaron ideas surgidas durante retroalimentación para realizar un video titulado "Actividades pedagógicas de socialización acerca de amenazas volcánicas en instituciones educativas del municipio de Cumbal Nariño" en el cual el autor HGC describe y explica diversos aspectos de la geografía, la geología y los volcanes de la región, interrelacionando conocimiento científico y ancestral y utilizando terminología y expresiones conocidas y accesibles a la comunidad indígena y campesina. Este video tiene una duración de 40 minutos y está a disposición de interesados previa solicitud a los autores. Finalmente se menciona que un resultado importante de todo el proceso fue el escrito titulado "Actividades pedagógicas acerca de amenaza volcánica en instituciones educativas del municipio de Cumbal" (Cuaical, 2017), que sirvió como base para este artículo. 
TABLA 1. Información relevante a cada visita de socialización.

\begin{tabular}{|c|c|c|c|c|}
\hline $\begin{array}{l}\text { Fecha } \\
(2017)\end{array}$ & IE & Audiencia y nivel & Temas socializados & Observaciones \\
\hline Febrero 17 & Divino Niño Jesús & $\begin{array}{l}35 \text { estudiantes de } \\
\text { grados Sexto a } \\
\text { Once }\end{array}$ & $\begin{array}{l}\text { Introducción } \\
\text { a geología y } \\
\text { vulcanología. } \\
\text { Amenazas volcánicas }\end{array}$ & $\begin{array}{l}\text { Se describieron } \\
\text { experiencias de } \\
\text { afectación por } \\
\text { actividad volcánica }\end{array}$ \\
\hline Febrero 20 & José Antonio Llorente & $\begin{array}{l}60 \text { estudiantes de } \\
\text { grados: Sexto a } \\
\text { Once. }\end{array}$ & $\begin{array}{l}\text { Métodos de } \\
\text { investigación en } \\
\text { geología, escalas y } \\
\text { contextos, minerales, } \\
\text { rocas y depósitos. } \\
\text { Amenazas volcánicas }\end{array}$ & $\begin{array}{l}\text { Se usaron apoyos } \\
\text { audiovisuales: Fotos, } \\
\text { mapa geológico } \\
\text { (plancha 447-Ipiales) }\end{array}$ \\
\hline $\begin{array}{l}\text { Marzo } 14 \\
\text { y } 15\end{array}$ & $\begin{array}{l}\text { Técnica Agropecuaria } \\
\text { Indígena de Panán }\end{array}$ & $\begin{array}{l}150 \text { estudiantes de } \\
\text { grados Octavos a } \\
\text { Onces. }\end{array}$ & $\begin{array}{l}\text { Amenazas volcánicas, } \\
\text { ejemplos a nivel } \\
\text { mundial. Conceptos de } \\
\text { riesgo, vulnerabilidad }\end{array}$ & $\begin{array}{l}\text { Énfasis en contexto } \\
\text { local. Uso de carteleras } \\
\text { y mapas de amenaza de } \\
\text { los volcanes Chiles y } \\
\text { Cumbal }\end{array}$ \\
\hline Marzo 30 & $\begin{array}{l}\text { Técnica Agropecuaria } \\
\text { Indígena CUMBE }\end{array}$ & $\begin{array}{l}25 \text { estudiantes de } \\
\text { grados Décimo y } \\
\text { Once. }\end{array}$ & $\begin{array}{l}\text { Teorías del origen de } \\
\text { la Tierra y Universo. } \\
\text { Estructura terrestre, } \\
\text { vulcanismo, procesos y } \\
\text { productos volcánicos. } \\
\text { El SNPAD la UNGRD }\end{array}$ & $\begin{array}{l}\text { Contexto local } \\
\text { (mención de sitios } \\
\text { bien conocidos en los } \\
\text { alrededores) }\end{array}$ \\
\hline Abril 6 & Nuestro Señor del Río & $\begin{array}{l}35 \text { estudiantes de } \\
\text { Grados Décimos }\end{array}$ & $\begin{array}{l}\text { Origen y edad } \\
\text { de la Tierra y } \\
\text { Universo, avances } \\
\text { de la vulcanología, } \\
\text { amenazas volcánicas, } \\
\text { prevención, } \\
\text { emergencia, } \\
\text { reconstrucción }\end{array}$ & $\begin{array}{l}\text { A petición del señor } \\
\text { rector, Ignacio Palma, } \\
\text { se trataron temas } \\
\text { relacionados a gestión } \\
\text { del riesgo de desastres }\end{array}$ \\
\hline Abril 12 & $\begin{array}{l}\text { Agroecológica Sagrado } \\
\text { Corazón de Jesús }\end{array}$ & $\begin{array}{l}100 \text { estudiantes de } \\
\text { Grados: Quinto a } \\
\text { Once. }\end{array}$ & $\begin{array}{l}\text { Amenazas volcánicas, } \\
\text { experiencia de Armero } \\
\text { (1985) }\end{array}$ & $\begin{array}{l}\text { A petición del señor } \\
\text { rector, Marín Chirán, } \\
\text { se trataron temas } \\
\text { relacionados a gestión } \\
\text { del riesgo de desastres. } \\
\text { Se siguió a Saavedra } \\
\text { \& Jaramillo (1995) } \\
\text { y documentos del } \\
\text { Ministerio del Interior }\end{array}$ \\
\hline Abril 24 & $\begin{array}{l}\text { Yo Reinaré, vereda } \\
\text { Boyera }\end{array}$ & $\begin{array}{l}30 \text { estudiantes de } \\
\text { Grado Décimo y } \\
\text { Once }\end{array}$ & $\begin{array}{l}\text { Vulcanología, } \\
\text { ejemplos locales, } \\
\text { nacionales e } \\
\text { internacionales }\end{array}$ & $\begin{array}{l}\text { Estudio del mapa de } \\
\text { Amenaza del volcán } \\
\text { Cumbal }\end{array}$ \\
\hline Abril 27 & San Juan de Mayasquer & $\begin{array}{l}35 \text { estudiantes de } \\
\text { grados Décimo y } \\
\text { once }\end{array}$ & $\begin{array}{l}\text { Edad y origen de la } \\
\text { Tierra y Universo, } \\
\text { vulcanismo y } \\
\text { extinciones bióticas. } \\
\text { Amenazas volcánicas } \\
\text { y gestión del riesgo }\end{array}$ & $\begin{array}{l}\text { La conferencia sirvió } \\
\text { como motivación } \\
\text { para continuar hacia } \\
\text { la vida universitaria y } \\
\text { profesional }\end{array}$ \\
\hline
\end{tabular}




\begin{tabular}{|c|c|c|}
\hline Fase (momento) & Acciones (técnicas) & Participantes \\
\hline 1. Selección de IE. & $\begin{array}{c}\text { Salidas de campo, } \\
\text { contactos iniciales con } \\
\text { directivos. }\end{array}$ & $\begin{array}{l}\text { HGC, rectores y profesores } \\
\text { de IE. }\end{array}$ \\
\hline \multicolumn{3}{|c|}{ \ } \\
\hline $\begin{array}{l}\text { 2. Planeación para } \\
\text { actividad pedagógica. }\end{array}$ & $\begin{array}{l}\text { Comunicación escrita a } \\
\text { directivos, detalles acerca } \\
\text { de la actividad, establecer } \\
\text { fecha y solicitar temas } \\
\text { especiales. }\end{array}$ & $\begin{array}{c}\text { HGC, profesores, y } \\
\text { encargados de logística en } \\
\text { la IE. }\end{array}$ \\
\hline \multicolumn{3}{|c|}{ 飞 } \\
\hline $\begin{array}{l}\text { 3. Preparación de } \\
\text { materiales y actividades. }\end{array}$ & $\begin{array}{l}\text { Selección de temas, } \\
\text { organización de } \\
\text { presentación, planeación } \\
\text { de tiempos, selección de } \\
\text { ayudas audiovisuales. }\end{array}$ & HGC. \\
\hline \multicolumn{3}{|c|}{ 亿 } \\
\hline $\begin{array}{l}\text { 4. Realización de } \\
\text { conferencia y taller. }\end{array}$ & $\begin{array}{c}\text { Presentación inicial, } \\
\text { agradecimientos, } \\
\text { conferencia, actividad } \\
\text { práctica. }\end{array}$ & $\begin{array}{l}\text { HGC, estudiantes, } \\
\text { profesores. }\end{array}$ \\
\hline
\end{tabular}

FIGURA 6. Esquema que resume el diseño de las actividades de socialización.

\section{DISCUSIÓN}

Las visitas e interacciones con la comunidad educativa del municipio de Cumbal permitieron evidenciar interés en los temas centrales relacionados a volcanes, amenazas y conformación geológica del territorio, pero también se observó fuerte influencia de la cosmovisión y las ideas ancestrales. Por tanto, se realizó un esfuerzo por contextualizar en términos de lugares tradicionales que incluso representan valores culturales, místicos y sagrados como cuerpos de agua cercanos, fuentes termales, afloramientos o rocas notables (Piedra de Los Machines, Piedra del Guacamullo, Piedra de Cumbaltar) (FIGURA 7) y por supuesto, los edificios volcánicos de la región (FIGURAS 2, 3 y 4). Aunque el objetivo principal de cada visita incluía presentar temas específicos de volcanes y sus amenazas, en cada una de las instituciones visitadas existían intereses variables acerca de tópicos adicionales que fueron tratados en la medida que el tiempo y la logística lo permitían. En varias ocasiones se recibieron solicitudes, previas a las visitas, acerca de temáticas de interés por parte de los estudiantes o sus profesores y esto permitió planear y orientar, hasta cierto punto, las interacciones. Esta dinámica, que involucró interacciones y retroalimentaciones e hizo de los participantes actores muy importantes en el desarrollo de la experiencia (Acevedo, 2008), introdujo variabilidad en el desarrollo de cada una de las visitas y aunque existía una planeación previa, ajustada a ciertos temas, fue necesario elaborar y extender hacia temáticas que surgían de acuerdo a diferentes expectativas y necesidades particulares o institucionales. Por ejemplo, en dos instituciones fue pertinente el uso de material especializado para guiar discusiones acerca de gestión del riesgo de desastres (TABLA 1), mientras que en otras el interés giró en torno al significado de sitios tradicionales y la cosmovisión del territorio. Desde esa perspectiva, se reconoció en esta experiencia que el autor HGC se identificó con muchas de las diferentes visiones y conceptos y su formación como Geólogo posibilitó la unión de los conocimientos de lo científico y lo ancestral. 

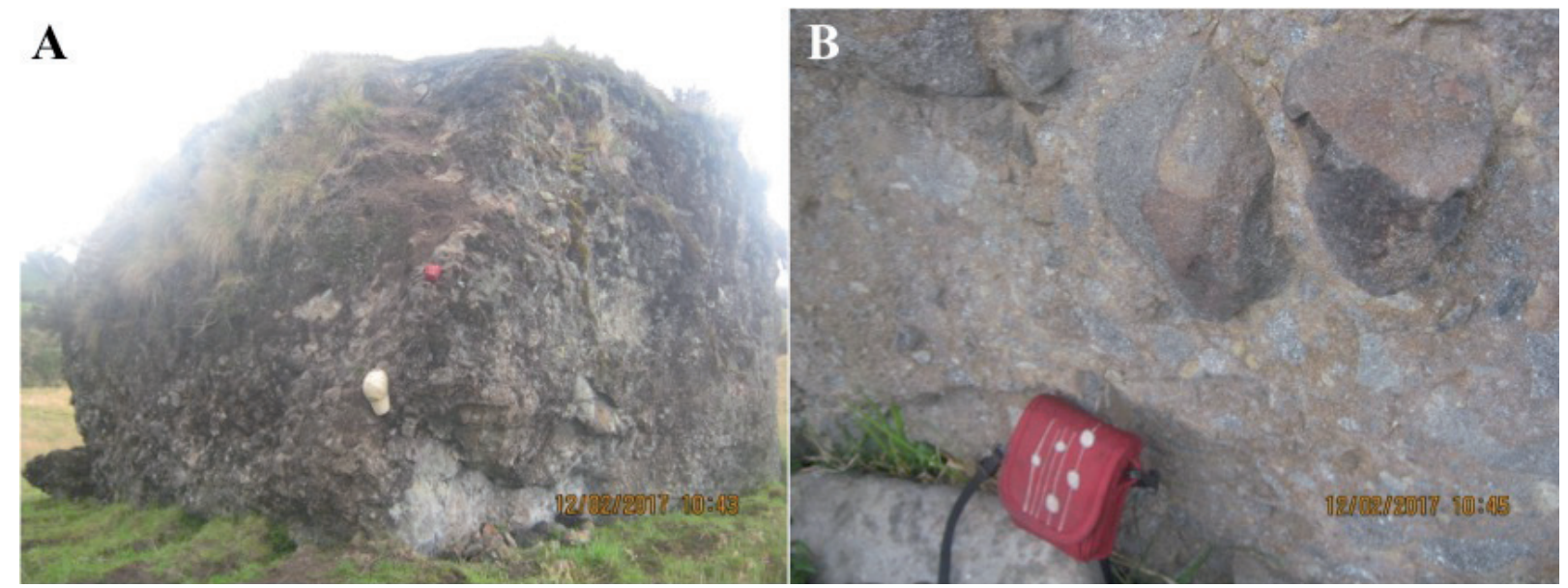

FIGURA 7. A. La Piedra del Guacamullo, en el sector Pedregal de la Vereda Tasmag. B. Detalle del megabloque donde se observan clastos de lava angulosos en matriz de ceniza y lapilli. Origen incierto, pero podría corresponder a un depósito de flujo de bloques y ceniza. De otra parte, la tradición ancestral señala a esta Piedra como sitio de origen de las comunidades que habitan la región.

Aunque se respondieron muchas inquietudes, también surgieron nuevos interrogantes y discusiones acerca de la concepción que tienen los estudiantes y etnoeducadores acerca del territorio y el universo. Algunos de estos tópicos pueden parecer alejados del tema central del vulcanismo, pero inevitablemente surgen e influencian el desarrollo de cualquier tipo de actividad científica, social, humanitaria o económica en los territorios de la denominada Nación Pasto. Dado que este aspecto ha sido constantemente reportado por otros que han sido participantes de actividades de socialización en la región, es importante dar idea, aunque sea somera, de la dimensión que rodea el trabajo en algunas regiones de Colombia. A continuación, se presenta una lista concisa de las diferentes temáticas que comúnmente son planteadas:

- Vulnerabilidad e historia de dominación de las comunidades indígenas desde el momento de la llegada de los europeos a América.

- La globalización vista como amenaza a los fundamentos naturales de la vida humana, con crecimiento expansivo que se basa cada vez más en la explotación de recursos no renovables y por lo tanto en la expropiación de la naturaleza.

- El desarrollo económico, que en buena parte está basado en la extracción de recursos, ha destruido el equilibrio entre las comunidades indígenas, la madre territorio y el cosmos. En ese sentido es posible que iniciativas importantes de búsqueda de fuentes alternativas de energía limpia, como la energía geotérmica (que seguramente son en principio bien intencionadas) hayan generado rechazo y desconfianza o se han visto como una amenaza a la identidad o al territorio.

- No es suficiente el conocimiento científico, hay también necesidad de reciprocar a la madre tierra con ofrendas de comida (avíos), idea consignada en los discursos de un taita influyente: “...el volcán Chiles anda molesto y comenzó a temblar por la búsqueda de la geotermia. Los movimientos telúricos no los han podido calmar los científicos, sino cuando los mayores subimos con el avio a agradecer, a meditar, a rezar. Entonces se ha calmado" (Tarapués, 2016),

- La importancia de muchos mitos y leyendas (relacionados por ejemplo al origen de las diferentes comunidades que ocupan los territorios) y rasgos del paisaje (volcanes, montañas, rocas) (FIGURA 7) que están en la tradición oral y la cultura de la región y que son trazados hasta las enseñanzas del sabio Taita Juan Chiles en el siglo XVIII, una figura ancestral de máxima importancia (FIGURA 8). 


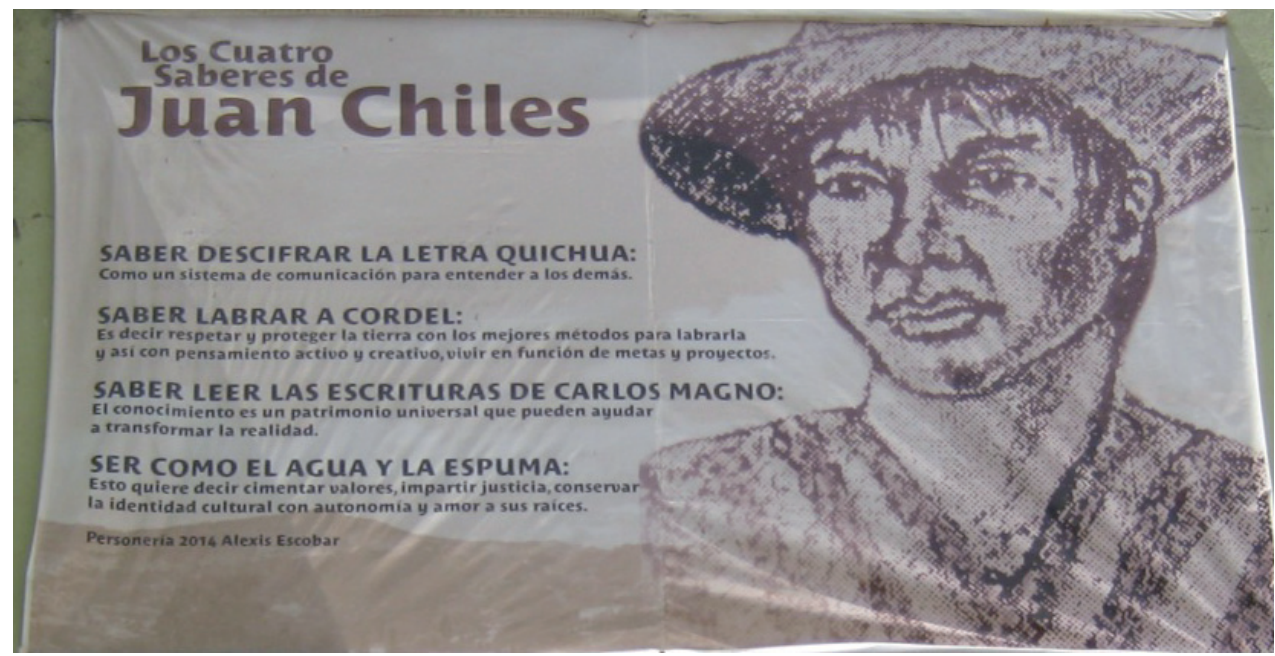

FIGURA 8. Pancarta con la imagen del sabio Taita Juan Chiles, desplegada en la IE Nuestro Señor del Río. Se enuncian los cuatro saberes: 1) Saber descifrar la letra Quichua (comunicación para entender a los demás); 2) Saber labrar a cordel (respetar y proteger la Tierra); 3) Saber leer las escrituras de Carlos Magno (el conocimiento, patrimonio universal, puede ayudar a transformar la realidad), 4) Ser como el agua y la espuma (valores, justicia, identidad cultural, autonomía y amor a las raíces).

Este tipo de ideas, que pueden no estar muy ajustadas a la ciencia o a la necesidad apremiante actual de generación de energía limpia, si constituyen una forma de conocimiento (Carbonelli et al., 2017) y tienen el mérito de ser el primer y quizá único conocimiento que estuvo al alcance de las comunidades durante muchas generaciones y han de ser tenidas en cuenta al emprender futuras actividades de investigación, de exploración de recursos o de desarrollo en la región. Por ello, durante esta experiencia, se siguió el ejemplo de otras regiones y se buscaron respuestas en la ciencia geológica (FIGURA 9) y en el conocimiento ancestral (Asociación Boliviana Para el Avance de la CienciaABAC, 2007), sobretodo manteniendo una actitud imparcial y a la vez una mirada crítica ante distintos escenarios geopolíticos y coyunturales que viven las comunidades indígenas. En la actualidad se reconoce que parte de las respuestas a estos temas están fundamentadas en interculturalidad y respeto por las diferencias (Beltrán, 2015) y se destaca la importancia que cobran la educación para la diversidad, la educación intercultural o la educación inclusiva (Aguado y Mata, 2017; Echeita, 2006). También se aprende que es central profundizar en el conocimiento de las comunidades indígenas e incorporar este conocimiento en futuras actividades de investigación, exploración de recursos o de desarrollo.

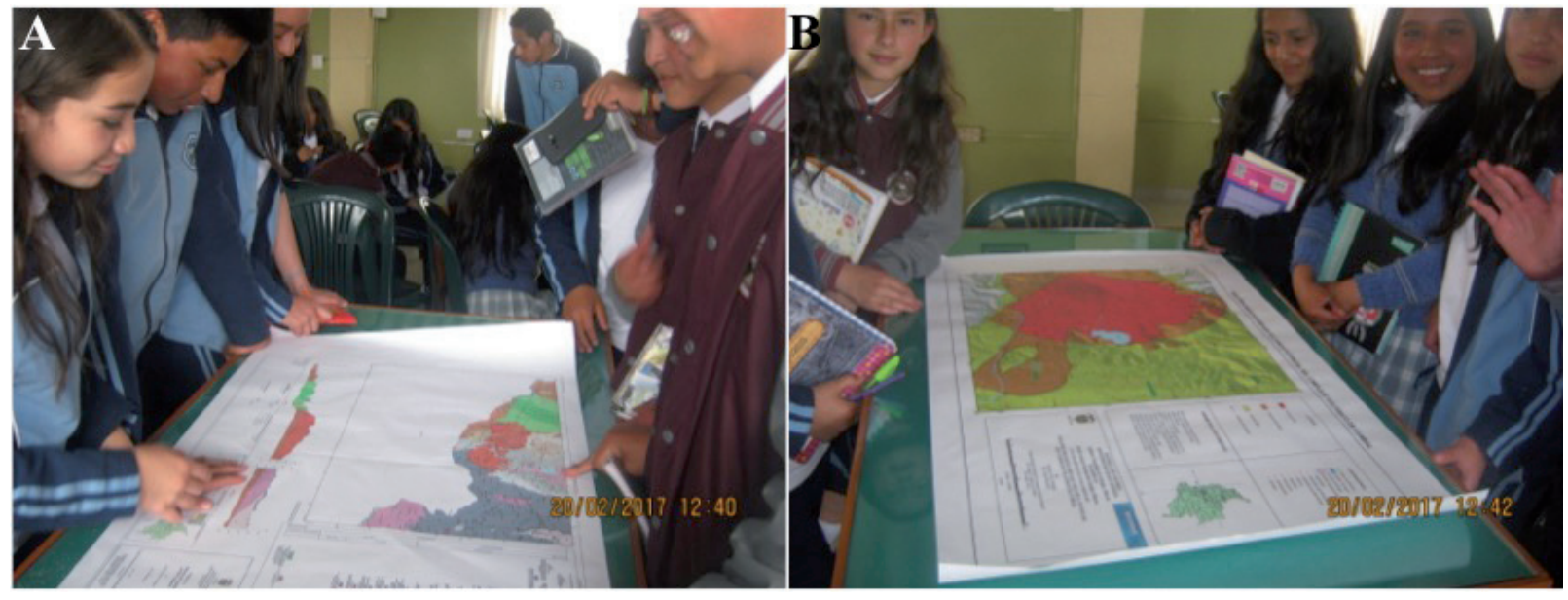

FIGURA 9. Estudiantes de la IE José Antonio Llorente participando en actividades durante una de las conferencias. A. Observan el mapa geológico 447 Ipiales (Velásquez y Parra, 2002). B. Estudio del mapa de amenaza del CVC (INGEOMINAS, 2000). 


\section{CONCLUSIONES}

La experiencia documentada en este artículo giró en torno a socialización sobre amenazas volcánicas en ocho instituciones educativas del municipio de Cumbal, Departamento de Nariño, Colombia. Se confirmó que ambos tipos de saberes: científico y ancestral son necesarios y que son muy bien recibidos por los participantes cuando el socializador pertenece a la comunidad. Desde las áreas de experiencia de los autores (la geología y la vulcanología) se aprendió que, para que la misión del geólogo o del vulcanólogo trascienda en estos entornos (comunidades indígenas y campesinas), es crucial el trabajo interdisciplinario y el conocimiento acerca de lo ancestral, lo místico y lo sagrado. También se estableció firmemente que en todo tipo de experiencias de socialización es necesario que los participantes mantengan una actitud de mente abierta al estar expuestos a múltiples diferencias que surgen precisamente por la variabilidad en audiencias.

\section{RECOMENDACIONES}

- Los rectores de las diferentes instituciones etno-pedagógicas recomendaron que durante las conferencias y actividades no se rompiera el esquema tradicional de la concepción del territorio, el cosmos y los fenómenos naturales. En concordancia con esto, la intención no ha sido ir en contra de la identidad cultural sino más bien dar una explicación alternativa desde la cual el método científico puede aportar.

- Algunos estudiantes solicitaron que se vuelvan a compartir conferencias relacionadas con algunos temas geológicos como minería, energía geotérmica, arqueología. En lo posible se continuará con estas actividades entre los estudiantes y la comunidad.

- Se recomienda fortalecer los conocimientos de Física y Química entre los estudiantes de nuestras comunidades educativas.

- Es fundamental realizar muchas salidas de campo a sitios sagrados, espirituales y cosmo-referenciales (a las lagunas, ríos, fuentes termales, Piedra de Guacamullo, Piedra de Cumbaltar, Piedra de Machines) y a las estructuras volcánicas.

- Es importante seguir profundizando más en el conocimiento y la interpretación de los sitios cosmo-referenciales, lugares sagrados y espirituales del Pueblo Pasto con la ayuda de profesionales en ciencias como astronomía, antropología, arqueología, biología, etc.

- Se recomienda fomentar, generar y apoyar propuestas con fines turísticos que serían de gran ayuda para muchas familias de la región y afianzarían la apropiación del territorio.

\section{AGRADECIMIENTOS}

Los autores agradecen a los integrantes de intituciones educativas del municipio de Cumbal que participaron en las experiencias de socialización y apoyaron el desarrollo de este trabajo. Los comentarios y sugerencias de la Dra. Mabel Padlog (Centro Universitario de Ciencias Sociales y HumanidadesCUCSH, Universidad de Guadalajara, México) y la Dra. Nelly Aguilar Rueda (Unidades Tecnológicas de Santander) ayudaron a mejorar la calidad del artículo.

\section{REFERENCIAS}

Acevedo, M. (2008). La metáfora de los escenarios de educación popular. Magisterio, 33, 24-31.

Aguado, T., y Mata, P. (2017). Educación intercultural. Madrid: Universidad Nacional de Educación a Distancia.

Alcaldía de Cumbal-Nariño. (2012). Sitio oficial de Cumbal en Nariño, Colombia Indicadores del Municipio. Consultado el 24 de mayo de 2017. http://www.cumbal-narino.gov. co/indicadores_anuales.shtml $? \mathrm{apc}=\mathrm{bexx}-1$ $\& x=2982899$ \# educacion.

Asociación Boliviana Para el Avance de la CienciaABAC (2007). Primer Seminario-Taller-Ciencia, Tecnología y Conocimiento Ancestral en Bolivia. La Paz, Bolivia, 30 de noviembre al 2 de diciembre de 2006.

Beltrán, J. (2015). La interculturalidad. Barcelona: Editorial UOC.

Bermúdez, C. (2018). Lógica práctica y lógica teórica en la sistematización de experiencias educativas. Pedagogía y Saberes, 48, 141-151.

Carbonelli, M., Cruz, J., y Irrazábal, G. (2017). Introducción al conocimiento científico y a la 
metodología de la investigación. Buenos Aires: Universidad Nacional Arturo Jauretche.

Cuaical, H.G. (2017). Actividades pedagógicas acerca de amenaza volcánica en instituciones educativas del Municipio de Cumbal. Tesis, Universidad Nacional de Colombia, Bogotá, Colombia.

Echeita, G. (2006). Educación para la inclusión o educación sin exclusiones. Madrid: Ediciones Narcea.

El Diario. (2015). La lección no aprendida de Armero. Consultado el 27 de Mayo de 2017. http://www. eldiario.com.co.

Espíndola, J.M., Macías, J.L., Tilling, R.I., and Sheridan, M.F. (2000). Volcanic history of El Chichón Volcano (Chiapas, Mexico) during the Holocene, and its impact on human activity. Bulletin of Volcanology, 62(2), 90-104.

Gil, P.C., y Sánchez, J.J. (2014). Los volcanes en Colombia. Bogotá: Universidad Nacional de Colombia.

Gómez, J., Montes, N.E., Nivia, A., y Diederix, H. (2015). Mapa geológico de Colombia 2015. Escala 1:1.000.000. Servicio Geológico Colombiano.

Gómez, D., y Narváez, P. (2017). Experiencia del Servicio Geológico Colombiano con comunidades educativas indígenas en el Municipio de Cumbal, Departamento de Nariño, como aporte a la apropiación social del conocimiento geocientífico. XVI Congreso Colombiano de Geología. Santa Marta, Colombia.

Hall, M. (1990). Chronology of the principal scientific and governmental actions leading up to the November 13, 1985 eruption of Nevado del Ruiz, Colombia. Journal of Volcanology and Geothermal Research, 42(1-2), 101-115. doi: 10.1016/0377-0273(90)90072-N.

INGEOMINAS. (2000). Atlas de amenaza volcánica en Colombia. Bogotá: INGEOMINAS.

Jara, O. (2008). Sistematización de experiencias: Un concepto enraizado en la realidad latinoamericana. Magisterio, 33, 8-19.
Latorre, E. (1987). Los desastres naturales. El caso de Armero: Un enfoque de sistemas. Heurística, 2(1), 9-21.

Lécuyer, F., y Cheminée, J.L. (2010). Volcanes. Madrid: Ediciones Tikal.

Macedo, L. (2012). Preparación ante emergencias de origen volcánico. Una guía para la reducción del riesgo volcánico en Perú. Boletín 49, Serie C, Geodinámica e Ingeniería Geológica. INGEMMET, $125 \mathrm{p}$.

Mojica, J., Colmenares, F., Villarroel, C., Macía, C., y Moreno, M. (1985). Características del flujo de lodo ocurrido el 13 de Noviembre de 1985 en el valle de Armero (Tolima, Colombia): Historia y comentarios de los flujos de 1595 y 1845. Geología Colombiana, 14, 107-140.

Macías, J.L., Arce, J.L., Mora, J.C., Espíndola, J.M., Saucedo, R., and Manetti, P. (2003). A 550-yearold Plinian eruption at El Chichón Volcano, Chiapas, Mexico: Explosive volcanism linked to reheating of the magma reservoir. Journal of Geophysical Research: Solid Earth, 108(B12), 2569, 1-18. doi: 10.1029/2003JB002551.

Parra, E., Cepeda, H., y Thouret, J.C. (1986). Mapa actualizado de amenaza volcánica potencial del Nevado del Ruiz. INGEOMINAS, Bogotá.

Parra, E., and Cepeda, H. (1990). Volcanic hazard maps of the Nevado del Ruiz volcano, Colombia. Journal of Volcanology and Geothermal Research, 42(1-2), 117-127. doi: 10.1016/03770273(90)90073-O.

Pierson, T.C., Janda, R.J., Thouret, J.C., and Borrero, C.A. (1990). Perturbation and melting of snow and ice by the 13 November 1985 eruption of Nevado del Ruiz, Colombia, and consequent mobilization, flow and deposition of lahars. Journal of Volcanology and Geothermal Research, 41(1-4), 17-66. doi: 10.1016/0377-0273(90)90082-Q.

Scarth, A. (2002). La catastrophe: Mount Pelée and the destruction of Saint-Pierre. Martinique: Terra Publishing, 256p. 
Soto, G.J. (2012). Preparación de mapas de peligros volcánicos y restricción de uso de la tierra en el volcán Turrialba. Informe Final. Fundación de la Universidad de Costa Rica para la Investigación FUNDEVI. San Jose, Costa Rica.

Tarapués, E.F. (2016). Los veintidós principios rectores de los indígenas Pastos y el principio de la ritualidad. Conferencia, auditorio Museo del Oro Banrepcultural, Banco de la República Colombia. abril 7. Consultado el 22 de septiembre de 2017. https://www.youtube.com/ watch? $v=2 h 0 h e M k D f F Y$.

Tilling, R.I. (2009). El Chichón's “surprise” eruption in 1982: Lessons for reducing volcano risk. Geofísica Internacional, 48(1), 3-19.

UNGRD y Presidencia de la República (2015). Audiencia Pública de Rendición de Cuentas. Unidad Nacional para la Gestión del Riesgo de Desastres-Colombia. 125p.
Velásquez, M., y Parra, E. (2002). Geología de las planchas 447-Ipiales y 447 bis-Tallambí, Memoria explicativa, Escala 1:100.000. INGEOMINAS, $118 \mathrm{p}$.

Voight, B. (1990). The 1985 Nevado del Ruiz volcano catastrophe: anatomy and retrospection. Journal of Volcanology and Geothermal Research, 42(3-4), 349-386. doi: 10.1016/0377-0273(90)90027-D.

\begin{tabular}{c}
\hline \hline Hermes G. Cuaical \\
ORCID: 0000-0002-7282-8793 \\
John J. Sánchez \\
ORCID: 0000-0002-9024-7554 \\
\hline \hline
\end{tabular}

Trabajo recibido: octubre 13 de 2017

Trabajo aceptado: mayo 17 de 2018 